\title{
Existence results for nonlinear boundary value problems with integral boundary conditions on an infinite interval
}

Fulya Yoruk* and Nuket Aykut Hamal

"Correspondence:

fulya.yoruk@ege.edu.tr

Department of Mathematics, Ege

University, Bornova, Izmir, 35100,

Turkey

\begin{abstract}
In this paper, by using fixed point theorems in a cone, the existence of one positive solution and three positive solutions for nonlinear boundary value problems with integral boundary conditions on an infinite interval are established.

MSC: 34B10; 39A10; 34B18; 45G10
\end{abstract}

Keywords: positive solutions; fixed point theorems; integral boundary conditions; infinite interval

\section{Introduction}

Consider the following boundary value problem with integral boundary conditions on the half-line of an infinite interval of the form

$$
\begin{aligned}
& \frac{1}{p(t)}\left(p(t) z^{\prime}(t)\right)^{\prime}+f\left(t, z(t), z^{\prime}(t)\right)=0, \quad t \in(0, \infty), \\
& a_{1} z(0)-b_{1} \lim _{t \rightarrow 0^{+}} p(t) z^{\prime}(t)=\int_{0}^{\infty} g_{1}(z(s)) \psi(s) d s, \\
& a_{2} \lim _{t \rightarrow+\infty} z(t)+b_{2} \lim _{t \rightarrow+\infty} p(t) z^{\prime}(t)=\int_{0}^{\infty} g_{2}(z(s)) \psi(s) d s,
\end{aligned}
$$

where $f \in \mathcal{C}((0, \infty) \times[0, \infty) \times \mathbb{R},[0, \infty)), f$ may be singular at $t=0 ; g_{1}, g_{2}:[0, \infty) \rightarrow[0, \infty)$ are continuous, nondecreasing functions and for $0 \leq t<\infty, z$ in a bounded set, $g_{1}(z)$, $g_{2}(z)$ are bounded; $\psi:[0, \infty) \rightarrow(0, \infty)$ is a continuous function with $\int_{0}^{\infty} \psi(s) d s<+\infty$; $p \in \mathcal{C}[0, \infty) \cap \mathcal{C}^{1}(0, \infty)$ with $p(t)>0$ on $(0,+\infty)$ and $\int_{0}^{+\infty} \frac{d s}{p(s)}<+\infty ; a_{1}+a_{2}>0, b_{i}>0$ for $i=1,2$ with $D=a_{2} b_{1}+a_{1} b_{2}+a_{1} a_{2} B(0, \infty)>0$ in which $B(t, s)=\int_{t}^{s} \frac{d s}{p(s)}$.

Boundary value problems on an infinite interval appear often in applied mathematics and physics. There are many papers concerning the existence of solutions on the half-line for boundary value problems; see [1-5] and the references therein.

At the same time, boundary value problems with integral boundary conditions are of great importance and are an interesting class of problems. They constitute two, three, multi-point, and nonlocal boundary value problems as special cases. For an overview of the literature on integral boundary value problems, see [6-11] and the references therein.

\section{严 Springer}

(c) 2012 Yoruk and Hamal; licensee Springer. This is an Open Access article distributed under the terms of the Creative Commons Attribution License (http://creativecommons.org/licenses/by/2.0), which permits unrestricted use, distribution, and reproduction in any medium, provided the original work is properly cited. 
Yan Sun et al. [4] studied the existence of positive solutions for singular boundary value problems on the half-line for the following Sturm-Liouville boundary value problem:

$$
\begin{aligned}
& \frac{1}{p(t)}\left(p(t) z^{\prime}(t)\right)^{\prime}+\mu f\left(t, z(t), z^{\prime}(t)\right)=0, \quad t \in(0, \infty), \\
& a_{1} z(0)-b_{1} \lim _{t \rightarrow 0^{+}} p(t) z^{\prime}(t)=0, \\
& a_{2} \lim _{t \rightarrow+\infty} z(t)+b_{2} \lim _{t \rightarrow+\infty} p(t) z^{\prime}(t)=0,
\end{aligned}
$$

where $\mu$ is a positive parameter; $f$ is a continuous, non-negative function and may be singular at $t=0 ; p \in \mathcal{C}[0, \infty) \cap \mathcal{C}^{1}(0, \infty)$ with $p(t)>0$ on $(0,+\infty)$ and $\int_{0}^{+\infty} \frac{d s}{p(s)}<+\infty ; a_{i}, b_{i} \geq 0$ for $i=1,2$. Wang et al. [5] investigated the existence theorems for the boundary value problem given by

$$
\begin{aligned}
& \left(p(t) z^{\prime}(t)\right)^{\prime}+\lambda\left(f(t, z(t))-k^{2} z(t)\right)=0, \quad t \in(0, \infty), \\
& \alpha_{1} z(0)-\beta_{1} \lim _{t \rightarrow 0^{+}} p(t) z^{\prime}(t)=0, \\
& \alpha_{2} \lim _{t \rightarrow+\infty} z(t)+\beta_{2} \lim _{t \rightarrow+\infty} p(t) z^{\prime}(t)=0,
\end{aligned}
$$

where $f$ is a continuous, non-negative function and may be singular at $t=0 ; p \in \mathcal{C}[0, \infty) \cap$ $\mathcal{C}^{1}(0, \infty)$ with $p(t)>0$ on $(0,+\infty)$ and $\int_{0}^{+\infty} \frac{d s}{p(s)}<+\infty ; \alpha_{i} \beta_{i} \geq 0$ for $i=1,2$. Also, Feng [11] considered the following boundary value problem with integral boundary conditions on a finite interval:

$$
\begin{aligned}
& \left(g(t) z^{\prime}(t)\right)^{\prime}+w(t) f(t, z(t))=0, \quad t \in(0,1), \\
& a z(0)-b \lim _{t \rightarrow 0^{+}} g(t) z^{\prime}(t)=\int_{0}^{1} h(s) z(s) d s, \\
& a z(1)-b \lim _{t \rightarrow 1^{-}} g(t) z^{\prime}(t)=\int_{0}^{1} h(s) z(s) d s,
\end{aligned}
$$

where $a, b>0 ; g \in \mathcal{C}^{1}([0,1],[0, \infty)), w \in L_{p}[0,1], 1 \leq p \leq \infty$, and $h \in L_{1}[0,1]$ are symmetric functions; $f:[0,1] \times[0, \infty) \rightarrow[0, \infty)$ is continuous. The author obtained the existence of symmetric positive solutions by using the fixed point index theory in cones.

Motivated by the above works, we consider the existence of one and three positive solutions for the BVP (1.1), (1.2). However, to our knowledge, although various existence theorems are obtained for Sturm-Liouville boundary value problems with homogeneous boundary conditions, problems with nonhomogeneous boundary conditions, especially integral boundary conditions on an infinite interval have rarely been considered. Therefore, our boundary conditions are more general.

The rest of the paper is organized as follows. In Section 2, we present some necessary lemmas that will be used to prove our main results. In Section 3, we apply the Schauder fixed point theorem to get the existence of at least one positive solution for the nonlinear boundary value problem (1.1) and (1.2). In Section 4, we use the Leggett-Williams fixed point theorem [12] to get the existence of at least three positive solutions for the nonlinear boundary value problem (1.1) and (1.2). 


\section{Preliminaries}

In this section, we will employ several lemmas to prove the main results in this paper. These lemmas are based on the following BVP for $h \in \mathcal{C}((0, \infty))$ :

$$
\begin{aligned}
& \frac{1}{p(t)}\left(p(t) z^{\prime}(t)\right)^{\prime}+h(t)=0, \quad t \in(0, \infty), \\
& a_{1} z(0)-b_{1} \lim _{t \rightarrow 0^{+}} p(t) z^{\prime}(t)=\int_{0}^{\infty} g_{1}(z(s)) \psi(s) d s, \\
& a_{2} \lim _{t \rightarrow+\infty} z(t)+b_{2} \lim _{t \rightarrow+\infty} p(t) z^{\prime}(t)=\int_{0}^{\infty} g_{2}(z(s)) \psi(s) d s .
\end{aligned}
$$

Define $\varphi(t)$ and $\theta(t)$ to be the solutions of the corresponding homogeneous equation

$$
\frac{1}{p(t)}\left(p(t) z^{\prime}(t)\right)=0, \quad t \in(0, \infty)
$$

under the initial conditions,

$$
\begin{aligned}
& \theta(0)=b_{1}, \quad \lim _{t \rightarrow 0^{+}} p(t) \theta^{\prime}(t)=a_{1}, \\
& \lim _{t \rightarrow+\infty} \varphi(t)=b_{2}, \quad \lim _{t \rightarrow+\infty} p(t) \varphi^{\prime}(t)=-a_{2} .
\end{aligned}
$$

Using the initial conditions (2.4), we can deduce, from equation (2.3) for $\theta(t)$ and $\varphi(t)$, the following equations:

$$
\begin{aligned}
& \theta(t)=b_{1}+a_{1} \int_{0}^{t} \frac{d \tau}{p(\tau)}, \\
& \varphi(t)=b_{2}+a_{2} \int_{t}^{\infty} \frac{d \tau}{p(\tau)} .
\end{aligned}
$$

Let $G(t, s)$ be the Green function for $(2.1),(2.2)$ is given by

$$
G(t, s)=\frac{1}{D} \begin{cases}\theta(t) \varphi(s), & 0 \leq t \leq s<\infty \\ \theta(s) \varphi(t), & 0 \leq s \leq t<\infty\end{cases}
$$

where $\theta(t)$ and $\varphi(t)$ are given in (2.5) and (2.6) respectively.

Lemma 2.1 Suppose the conditions $\int_{0}^{+\infty} \frac{d s}{p(s)}<+\infty$ and $D>0$ hold. Then for any $h \in$ $\mathcal{C}((0, \infty), \mathbb{R})$, the $B V P(2.1),(2.2)$ has the unique solution

$$
z(t)=\int_{0}^{\infty} G(t, s) p(s) h(s) d s+\frac{\varphi(t)}{D} \int_{0}^{\infty} g_{1}(z(s)) \psi(s) d s+\frac{\theta(t)}{D} \int_{0}^{\infty} g_{2}(z(s)) \psi(s) d s,
$$

where $G(t, s)$ is given by $(2.7)$.

Furthermore, it is easy to prove the following properties of $G(t, s)$ :

(1) $G(t, s)$ is continuous on $[0,+\infty) \times[0,+\infty)$.

(2) For each $s \in[0,+\infty), G(t, s)$ is continuously differentiable on $[0,+\infty)$ except $t=s$.

(3) $\left.\frac{\partial G(t, s)}{\partial t}\right|_{t=s^{+}}-\left.\frac{\partial G(t, s)}{\partial t}\right|_{t=s^{-}}=-\frac{1}{p(s)}$. 
(4) $\left|G^{\prime}(t, s)\right| \leq \frac{c}{p(t)} G(s, s)$, for $t \in[0, \infty)$, where

$$
c=\frac{\max \left\{a_{1}, a_{2}\right\}}{\min \left\{b_{1}, b_{2}\right\}} .
$$

(5) For each $s \in[0,+\infty), G(t, s)$ satisfies the corresponding homogeneous BVP (i.e., $h(t) \equiv 0$ in the BVP $(2.1))$ on $[0,+\infty)$ except $t=s$.

(6) $0 \leq G(t, s) \leq G(s, s)$ for $t, s \in[0, \infty)$ and

$$
\bar{G}(s):=\lim _{t \rightarrow+\infty} G(t, s)=\frac{b_{2}}{D} \theta(s) \leq G(s, s)<+\infty .
$$

(7) For any $t \in[a, b] \subset(0, \infty)$ and $s \in[0,+\infty)$, we have

$$
G(t, s) \geq \gamma_{0} G(s, s)
$$

where

$$
\gamma_{0}=\min \left\{\frac{b_{2}+a_{2} B(b, \infty)}{b_{2}+a_{2} B(0, \infty)}, \frac{b_{1}+a_{1} B(0, a)}{b_{1}+a_{1} B(0, \infty)}\right\}
$$

Obviously, $0<\gamma_{0}<1$.

It is convenient to list the following conditions which are to be used in our theorems:

$(\mathrm{H} 1) f \in \mathcal{C}((0, \infty) \times[0, \infty) \times \mathbb{R})$ and also, $u(t) h(x, y) \leq f(t, x, y) \leq v(t) h(x, y), 0<t<\infty$, where $h \in C([0, \infty) \times R,(0, \infty))$; and for $0 \leq t<\infty, x, y$ in a bounded set, $h(x, y)$ is bounded and $u, v:(0, \infty) \rightarrow(0, \infty)$ is continuous and may be singular at $t=0$; and also, there exists $0<k_{0}<1$ such that $u(t) \geq k_{0} v(t)$ for $t \in(0, \infty)$.

$(\mathrm{H} 2) g_{1}, g_{2}:[0, \infty) \rightarrow[0, \infty)$ are continuous, nondecreasing functions, and for $0 \leq t<$ $\infty, z$ in a bounded set, $g_{1}(z), g_{2}(z)$ are bounded.

$(\mathrm{H} 3) \psi:[0, \infty) \rightarrow(0, \infty)$ is a continuous function with $\int_{0}^{\infty} \psi(s) d s<+\infty$.

$(\mathrm{H} 4) \int_{0}^{\infty} G(s, s) p(s) v(s) d s<+\infty$ and $\int_{0}^{\infty} G(s, s) p(s) u(s) d s<+\infty$.

Consider the Banach space

$$
B=\left\{z \in \mathcal{C}^{1}[0,+\infty): \lim _{t \rightarrow+\infty} z(t)<\infty, \sup _{t \in[0, \infty)}\left|z^{\prime}(t)\right|<\infty\right\}
$$

with the norm $\|z\|=\max \left\{\sup _{t \in[0, \infty)}|z(t)|, \sup _{t \in[0, \infty)}\left|z^{\prime}(t)\right|\right\}$.

From the above assumptions, we can define an operator $A: \mathcal{B} \rightarrow \mathcal{B}$ by

$$
\begin{aligned}
A z(t)= & \int_{0}^{\infty} G(t, s) p(s) f\left(s, z(s), z^{\prime}(s)\right) d s+\frac{\varphi(t)}{D} \int_{0}^{\infty} g_{1}(z(s)) \psi(s) d s \\
& +\frac{\theta(t)}{D} \int_{0}^{\infty} g_{2}(z(s)) \psi(s) d s, \quad t \in(0, \infty),
\end{aligned}
$$

where $G(t, s)$ is given by $(2.7)$.

Lemma 2.2 ([13]) Let $\mathcal{B}$ be defined as before and $M \subset \mathcal{B}$. Then $M$ is relatively compact in $\mathcal{B}$ if the following conditions hold:

(a) $M$ is uniformly bounded in $\mathcal{B}$; 
(b) The functions belonging to $M$ are equicontinuous on any compact interval of $[0, \infty)$;

(c) The functions from $M$ are equiconvergent, that is, given $\epsilon>0$, there corresponds a $T(\epsilon)>0$ such that $|f(t)-f(+\infty)|<\epsilon$ for any $t \geq T(\epsilon)$ and $f \in M$.

Definition 2.1 An operator is called completely continuous if it is continuous and maps bounded sets into relatively compact sets.

\section{Existence of at least one positive solution}

In this section, we will apply the following Schauder fixed point theorem to get an existence of one positive solution.

Theorem 3.1 (Schauder fixed point theorem) Let $\mathcal{B}$ be a Banach space and $S$ be a nonempty bounded, convex, and closed subset of $\mathcal{B}$. Assume $A: \mathcal{B} \rightarrow \mathcal{B}$ is a completely continuous operator. If the operator $A$ leaves the set $S$ invariant, i.e., if $A(S) \subset S$, then $A$ has at least one fixed point in $S$.

For convenience, let us set

$$
\begin{aligned}
& m:=\min \{\varphi(\infty), \theta(0)\}, \\
& M:=\max \{\varphi(0), \theta(\infty)\},
\end{aligned}
$$

and

$$
B(R)=\frac{M}{D}\left[g_{1}(R)+g_{2}(R)\right] \int_{0}^{\infty} \psi(s) d s .
$$

Theorem 3.2 Assume conditions (H1)-(H4) are satisfied. In addition, let there exist a number $R>0$ such that

$$
\max \left\{1, \sup _{t \in[0, \infty)} \frac{c}{p(t)}\right\} \sup _{z_{1} \in[0, R], z_{2} \in[-R, R]} h\left(z_{1}, z_{2}\right)\left(\int_{0}^{\infty} G(s, s) p(s) v(s) d s\right)+B(R) \leq R
$$

where $c$ is defined by (2.8).

Then the BVP (1.1), (1.2) has at least one solution $z$ with

$$
0 \leq z(t) \leq R, \quad t \in[0, \infty)
$$

Proof Let $A: \mathcal{B} \rightarrow \mathcal{B}$ be the operator defined by (2.9). We claim that $A$ is a completely continuous operator. To justify this, we first show that $A: \mathcal{B} \rightarrow \mathcal{B}$ is well defined. Let $z \in \mathcal{B}$, then there exists $r_{0}>0$ such that $\|z\| \leq r_{0}$ and from conditions (H1) and (H2), we have

$$
\begin{aligned}
& S_{r_{0}}:=\sup \left\{h(x, y): 0 \leq x \leq r_{0},-r_{0} \leq y \leq r_{0}\right\}<+\infty \\
& T_{r_{0}}:=\sup \left\{g_{1}(x): 0 \leq x \leq r_{0}\right\}<+\infty
\end{aligned}
$$

and

$$
T_{r_{0}}^{\prime}:=\sup \left\{g_{2}(x): 0 \leq x \leq r_{0}\right\}<+\infty
$$


Let $t_{1}, t_{2} \in[0, \infty), t_{1}<t_{2}$, then

$$
\int_{0}^{\infty}\left|G\left(t_{1}, s\right)-G\left(t_{2}, s\right)\right| p(s) \nu(s) d s \leq 2 \int_{0}^{\infty} G(s, s) p(s) v(s) d s<+\infty .
$$

Hence, by the Lebesgue dominated convergence theorem and the fact that $G(t, s)$ is continuous on $t$, we have

$$
\begin{aligned}
& \left|(A z)\left(t_{1}\right)-(A z)\left(t_{2}\right)\right| \leq \int_{0}^{\infty}\left|G\left(t_{2}, s\right)-G\left(t_{1}, s\right)\right| p(s) f\left(s, z(s), z^{\prime}(s)\right) d s \\
& +\frac{\left|\theta\left(t_{2}\right)-\theta\left(t_{1}\right)\right|}{D} \int_{0}^{\infty} g_{2}(z(s)) \psi(s) d s \\
& +\frac{\left|\varphi\left(t_{2}\right)-\varphi\left(t_{1}\right)\right|}{D} \int_{0}^{\infty} g_{1}(z(s)) \psi(s) d s \\
& \leq \int_{0}^{\infty}\left|G\left(t_{2}, s\right)-G\left(t_{1}, s\right)\right| p(s) v(s) h\left(z(s), z^{\prime}(s)\right) d s \\
& +\frac{\left|\theta\left(t_{2}\right)-\theta\left(t_{1}\right)\right|}{D} \int_{0}^{\infty} g_{2}(z(s)) \psi(s) d s \\
& +\frac{\left|\varphi\left(t_{2}\right)-\varphi\left(t_{1}\right)\right|}{D} \int_{0}^{\infty} g_{1}(z(s)) \psi(s) d s \\
& \leq S_{r_{0}} \int_{0}^{\infty}\left|G\left(t_{2}, s\right)-G\left(t_{1}, s\right)\right| p(s) v(s) d s \\
& +\frac{1}{D} \max \left\{\left|\varphi\left(t_{2}\right)-\varphi\left(t_{1}\right)\right|,\left|\theta\left(t_{2}\right)-\theta\left(t_{1}\right)\right|\right\} \\
& \times \int_{0}^{\infty}\left[g_{1}(z(s))+g_{2}(z(s))\right] \psi(s) d s \\
& \leq S_{r_{0}} \int_{0}^{\infty}\left|G\left(t_{2}, s\right)-G\left(t_{1}, s\right)\right| p(s) v(s) d s \\
& +\frac{1}{D} \max \left\{\left|\varphi\left(t_{2}\right)-\varphi\left(t_{1}\right)\right|,\left|\theta\left(t_{2}\right)-\theta\left(t_{1}\right)\right|\right\}\left(T_{r_{0}}+T_{r_{0}}^{\prime}\right) \int_{0}^{\infty} \psi(s) d s \\
& \rightarrow 0 \quad \text { as } t_{1} \rightarrow t_{2} \text {. }
\end{aligned}
$$

Also, by (H1) and (H2), we get

$$
\begin{aligned}
\left|(A z)^{\prime}\left(t_{1}\right)-(A z)^{\prime}\left(t_{2}\right)\right| \leq & \frac{a_{2}}{D}\left|\frac{1}{p\left(t_{1}\right)}-\frac{1}{p\left(t_{2}\right)}\right| \int_{0}^{t_{1}} \theta(s) p(s) f\left(s, z(s), z^{\prime}(s)\right) d s \\
& +\frac{a_{1}}{D p\left(t_{1}\right)} \int_{t_{1}}^{t_{2}} \varphi(s) p(s) f\left(s, z(s), z^{\prime}(s)\right) d s \\
& +\frac{a_{1}}{D}\left|\frac{1}{p\left(t_{1}\right)}-\frac{1}{p\left(t_{2}\right)}\right| \int_{t_{2}}^{\infty} \varphi(s) p(s) f\left(s, z(s), z^{\prime}(s)\right) d s \\
& +\frac{a_{2}}{D p\left(t_{2}\right)} \int_{t_{1}}^{t_{2}} \theta(s) p(s) f\left(s, z(s), z^{\prime}(s)\right) d s \\
& +\frac{a_{2}}{D}\left|\frac{1}{p\left(t_{1}\right)}-\frac{1}{p\left(t_{2}\right)}\right| \int_{0}^{\infty} g_{1}(z(s)) \psi(s) d s \\
& +\frac{a_{1}}{D}\left|\frac{1}{p\left(t_{1}\right)}-\frac{1}{p\left(t_{2}\right)}\right| \int_{0}^{\infty} g_{2}(z(s)) \psi(s) d s
\end{aligned}
$$




$$
\begin{aligned}
\leq & \frac{a_{2} S_{r_{0}}}{D}\left|\frac{1}{p\left(t_{1}\right)}-\frac{1}{p\left(t_{2}\right)}\right| \int_{0}^{t_{1}} \theta(s) p(s) v(s) d s \\
& +\frac{a_{1} S_{r_{0}}}{D p\left(t_{1}\right)} \int_{t_{1}}^{t_{2}} \varphi(s) p(s) v(s) d s \\
& +\frac{a_{1} S_{r_{0}}}{D}\left|\frac{1}{p\left(t_{1}\right)}-\frac{1}{p\left(t_{2}\right)}\right| \int_{t_{2}}^{\infty} \varphi(s) p(s) v(s) d s \\
& +\frac{a_{2} S_{r_{0}}}{D p\left(t_{2}\right)} \int_{t_{1}}^{t_{2}} \theta(s) p(s) v(s) d s \\
& +\frac{a_{2} T_{r_{0}}}{D}\left|\frac{1}{p\left(t_{1}\right)}-\frac{1}{p\left(t_{2}\right)}\right| \int_{0}^{\infty} \psi(s) d s \\
& +\frac{a_{1} T_{r_{0}}^{\prime}}{D}\left|\frac{1}{p\left(t_{1}\right)}-\frac{1}{p\left(t_{2}\right)}\right| \int_{0}^{\infty} \psi(s) d s \\
\rightarrow & 0 \quad \text { as } t_{1} \rightarrow t_{2} .
\end{aligned}
$$

So, $A z \in \mathcal{C}^{1}[0, \infty)$.

We can show that $A z \in \mathcal{B}$. Notice that

$$
\begin{aligned}
\lim _{t \rightarrow+\infty}(A z)(t)= & \int_{0}^{\infty} \bar{G}(s) p(s) f\left(s, z(s), z^{\prime}(s)\right) d s+\frac{\varphi(\infty)}{D} \int_{0}^{\infty} g_{1}(z(s)) \psi(s) d s \\
& +\frac{\theta(\infty)}{D} \int_{0}^{\infty} g_{2}(z(s)) \psi(s) d s<+\infty .
\end{aligned}
$$

In addition, we have

$$
\begin{aligned}
\left|(A z)^{\prime}(t)\right| \leq & \int_{0}^{\infty}\left|G^{\prime}(t, s)\right| p(s) v(s) h\left(z(s), z^{\prime}(s)\right) d s \\
& +\frac{a_{2}}{D p(t)} \int_{0}^{\infty} g_{1}(z(s)) \psi(s) d s+\frac{a_{1}}{D p(t)} \int_{0}^{\infty} g_{2}(z(s)) \psi(s) d s \\
\leq & \frac{c}{p(t)} \int_{0}^{\infty} G(s, s) p(s) v(s) h\left(z(s), z^{\prime}(s)\right) d s \\
& +\frac{\max \left\{a_{1}, a_{2}\right\}}{D p(t)} \int_{0}^{\infty}\left[g_{1}(z(s))+g_{2}(z(s))\right] \psi(s) d s \\
\leq & S_{r_{0}} \frac{c}{p(t)} \int_{0}^{\infty} G(s, s) p(s) v(s) d s \\
& +\left(T_{r_{0}}+T_{r_{0}}^{\prime}\right) \frac{\max \left\{a_{1}, a_{2}\right\}}{D p(t)} \int_{0}^{\infty} \psi(s) d s .
\end{aligned}
$$

Therefore, $\sup \left|(A z)^{\prime}(t)\right|<\infty$.

Hence, $A: \mathcal{B} \rightarrow \mathcal{B}$ is well defined.

Next, for any positive integer $m$, we denote the operator $A_{m}: \mathcal{B} \rightarrow \mathcal{B}$ by

$$
\begin{aligned}
\left(A_{m} z\right)(t)= & \int_{\frac{1}{m}}^{\infty} G(t, s) p(s) f\left(s, z(s), z^{\prime}(s)\right) d s+\frac{\varphi(t)}{D} \int_{0}^{\infty} g_{1}(z(s)) \psi(s) d s \\
& +\frac{\theta(t)}{D} \int_{0}^{\infty} g_{2}(z(s)) \psi(s) d s, \quad t \in[0, \infty),
\end{aligned}
$$


and prove that $A_{m}: \mathcal{B} \rightarrow \mathcal{B}$ is completely continuous for each $m \geq 1$. Let $\left\|z_{n}-z\right\| \rightarrow 0$ as $n \rightarrow+\infty$. We will show that $\left\|A_{m} z_{n}-A_{m} z\right\| \rightarrow 0$ as $n \rightarrow \infty$ in $\mathcal{B}$. We know that

$$
\begin{aligned}
& \int_{\frac{1}{m}}^{\infty} G(t, s) p(s)\left|f\left(s, z_{n}(s), z_{n}^{\prime}(s)\right)-f\left(s, z(s), z^{\prime}(s)\right)\right| d s \\
& \quad+\frac{\varphi(t)}{D} \int_{0}^{\infty}\left|g_{1}\left(z_{n}(s)\right)-g_{1}(z(s))\right| \psi(s) d s \\
& \quad+\frac{\theta(t)}{D} \int_{0}^{\infty}\left|g_{2}\left(z_{n}(s)\right)-g_{2}(z(s))\right| \psi(s) d s \\
& \leq \int_{\frac{1}{m}}^{\infty} G(s, s) p(s)\left(\left|f\left(s, z_{n}(s), z_{n}^{\prime}(s)\right)\right|+\left|f\left(s, z(s), z^{\prime}(s)\right)\right|\right) d s \\
& \quad+\frac{\varphi(0)}{D} \int_{0}^{\infty}\left|g_{1}\left(z_{n}(s)\right)-g_{1}(z(s))\right| \psi(s) d s \\
& \quad+\frac{\theta(\infty)}{D} \int_{0}^{\infty}\left|g_{2}\left(z_{n}(s)\right)-g_{2}(z(s))\right| \psi(s) d s \\
& \leq \int_{\frac{1}{m}}^{\infty} G(s, s) p(s)\left(v(s) h\left(z_{n}(s), z_{n}^{\prime}(s)\right)+v(s) h\left(z(s), z^{\prime}(s)\right)\right) d s \\
&+\frac{M}{D} \int_{0}^{\infty}\left|g_{1}\left(z_{n}(s)\right)-g_{1}(z(s))\right| \psi(s) d s \\
&+\frac{M}{D} \int_{0}^{\infty}\left|g_{2}\left(z_{n}(s)\right)-g_{2}(z(s))\right| \psi(s) d s \\
& \leq 2 S_{r^{*}} \int_{\frac{1}{m}}^{\infty} G(s, s) p(s) v(s) d s \\
&+\frac{M}{D} \int_{0}^{\infty}\left|g_{1}\left(z_{n}(s)\right)-g_{1}(z(s))\right| \psi(s) d s \\
&+\frac{M}{D} \int_{0}^{\infty}\left|g_{2}\left(z_{n}(s)\right)-g_{2}(z(s))\right| \psi(s) d s,
\end{aligned}
$$

where $r^{\prime \prime}>0$ is a real number such that $r^{\prime \prime} \geq \max _{n \in N}\left\{\|z\|,\left\|z_{n}\right\|\right\}, N$ is a natural number set, $S_{r^{*}}:=\sup \left\{h(x, y): 0 \leq x \leq r^{\prime \prime},-r^{\prime \prime} \leq y \leq r^{\prime \prime}\right\}<+\infty$.

Therefore, for any $\varepsilon>0$, there exists a sufficiently large $K_{0}\left(K_{0}>\frac{1}{m}\right)$ such that

$$
2 S_{r^{*}} \int_{K_{0}}^{\infty} G(s, s) p(s) v(s) d s<\frac{\varepsilon}{4}
$$

From the fact that $\left\|z_{n}-z\right\| \rightarrow 0$ as $n \rightarrow \infty$, we can see that for the above $\varepsilon>0$, there exists a sufficiently large natural number $N_{0} \in N$ such that if $n>N_{0}$, for any $s \in[0, \infty)$, we have

$$
\left|z_{n}(s)-z(s)\right| \leq\left\|z_{n}-z\right\|<\frac{\varepsilon}{4}\left(\frac{M}{D} \int_{\frac{1}{m}}^{K_{0}} G(k, k) p(k) d k\right)^{-1}\left(\int_{0}^{\infty} \psi(s) d s\right)^{-1}
$$

and

$$
\left|z_{n}^{\prime}(s)-z^{\prime}(s)\right| \leq\left\|z_{n}-z\right\|<\frac{\varepsilon}{4}\left(\frac{M}{D} \int_{\frac{1}{m}}^{K_{0}} G(k, k) p(k) d k\right)^{-1}\left(\int_{0}^{\infty} \psi(s) d s\right)^{-1} .
$$


On the other hand, by the continuity of $f(t, x, y)$, for the above $\varepsilon>0$, there exists a $\delta>0$, for any $t \in\left[\frac{1}{m}, K_{0}\right], x, x_{1} \in\left[0, r^{*}\right], y, y_{1} \in\left[-r^{*}, r^{*}\right]$ such that if $\left|x-x_{1}\right|<\delta,\left|y-y_{1}\right|<\delta$, we have

$$
\left|f(t, x, y)-f\left(t, x_{1}, y_{1}\right)\right|<\frac{\varepsilon}{4}\left(\int_{\frac{1}{m}}^{K_{0}} G(k, k) p(k) d k\right)^{-1} .
$$

From the fact that $\left\|z_{n}-z\right\| \rightarrow 0$ as $n \rightarrow \infty$, there exists a natural number $N_{1}>N_{0}$ such that when $n>N_{1}$, for any $s \in\left[\frac{1}{m}, K_{0}\right], z_{n}(s), z(s) \in\left[0, r^{*}\right], z_{n}^{\prime}(s), z^{\prime}(s) \in\left[-r^{*}, r^{*}\right]$ if $\left|z_{n}(s)-z(s)\right|<$ $\delta,\left|z_{n}^{\prime}(s)-z^{\prime}(s)\right|<\delta$, we have

$$
\left|f\left(s, z_{n}(s), z_{n}^{\prime}(s)\right)-f\left(s, z(s), z^{\prime}(s)\right)\right|<\frac{\varepsilon}{4}\left(\int_{\frac{1}{m}}^{K_{0}} G(k, k) p(k) d k\right)^{-1} .
$$

In addition to this, by the continuity of $g_{1}(x)$ and $g_{2}(x)$ on $[0, \infty)$, for the above $\varepsilon>0$, there exists a $\delta>0$ for any $t \in[0, \infty), x, x_{1} \in\left[0, r^{*}\right]$, such that if $\left|x-x_{1}\right|<\delta$, we have

$$
\left|g_{i}(x)-g_{i}\left(x_{1}\right)\right|<\frac{\varepsilon}{4}\left(\frac{M}{D} \int_{0}^{\infty} \psi(s) d s\right)^{-1}, \quad \text { for } i=1,2
$$

From $\left\|z_{n}-z\right\| \rightarrow 0$ as $n \rightarrow \infty$, there exists a natural number $N_{2}>N_{0}$ such that when $n>N_{2}$, for any $s \in[0, \infty), z_{n}(s), z(s) \in\left[0, r^{*}\right]$ if $\left|z_{n}(s)-z(s)\right|<\delta$, we have

$$
\left|g_{i}\left(z_{n}(s)\right)-g_{i}(z(s))\right|<\frac{\varepsilon}{4}\left(\frac{M}{D} \int_{0}^{\infty} \psi(s) d s\right)^{-1}, \quad \text { for } i=1,2 .
$$

Hence, if $N=\max \left\{N_{1}, N_{2}\right\}$, then

$$
\begin{aligned}
\left|\left(A_{m} z_{n}\right)(t)-\left(A_{m} z\right)(t)\right|= & \mid \int_{\frac{1}{m}}^{\infty} G(t, s) p(s) f\left(s, z_{n}(s), z_{n}^{\prime}(s)\right) d s \\
& -\int_{\frac{1}{m}}^{\infty} G(t, s) p(s) f\left(s, z(s), z^{\prime}(s)\right) d s \\
& +\frac{\varphi(t)}{D} \int_{0}^{\infty}\left[g_{1}\left(z_{n}(s)\right)-g_{1}(z(s))\right] \psi(s) d s \\
& +\frac{\theta(t)}{D} \int_{0}^{\infty}\left[g_{2}\left(z_{n}(s)\right)-g_{2}(z(s))\right] \psi(s) d s \mid \\
\leq & \int_{\frac{1}{m}}^{K_{0}} G(s, s) p(s)\left|f\left(s, z_{n}(s), z_{n}^{\prime}(s)\right)-f\left(s, z(s), z^{\prime}(s)\right)\right| d s \\
& +\int_{K_{0}}^{\infty} G(s, s) p(s)\left(f\left(s, z_{n}(s), z_{n}^{\prime}(s)\right)+f\left(s, z(s), z^{\prime}(s)\right)\right) d s \\
& +\frac{\varphi(0)}{D} \int_{0}^{\infty}\left|g_{1}\left(z_{n}(s)\right)-g_{1}(z(s))\right| \psi(s) d s \\
& +\frac{\theta(\infty)}{D} \int_{0}^{\infty}\left|g_{2}\left(z_{n}(s)\right)-g_{2}(z(s))\right| \psi(s) d s \\
\leq & \int_{\frac{1}{m}}^{K_{0}} G(s, s) p(s)\left|f\left(s, z_{n}(s), z_{n}^{\prime}(s)\right)-f\left(s, z(s), z^{\prime}(s)\right)\right| d s \\
& +2 S_{r^{\prime}} \int_{K_{0}}^{\infty} G(s, s) p(s) v(s) d s
\end{aligned}
$$




$$
\begin{aligned}
& +\frac{M}{D} \int_{0}^{\infty}\left|g_{1}\left(z_{n}(s)\right)-g_{1}(z(s))\right| \psi(s) d s \\
& +\frac{M}{D} \int_{0}^{\infty}\left|g_{2}\left(z_{n}(s)\right)-g_{2}(z(s))\right| \psi(s) d s \\
& =\varepsilon .
\end{aligned}
$$

Similarly, we can see that when $\left\|z_{n}-z\right\| \rightarrow 0$ as $n \rightarrow+\infty,\left|\left(A_{m} z_{n}\right)^{\prime}(t)-\left(A_{m} z\right)^{\prime}(t)\right| \rightarrow 0$ as $n \rightarrow+\infty$. This implies that $A_{m}: \mathcal{B} \rightarrow \mathcal{B}$ is a continuous operator for each natural number $m$.

Choose $P_{R}$ to be a bounded, convex, and closed set by

$$
\mathcal{P}_{\mathcal{R}}=\{z \in \mathcal{B}:\|z\| \leq R, z(t) \geq 0 \text { for each } t \in[0, \infty)\}
$$

We must show that there exists a positive constant $R$ such that for each $z \in P_{R}$, one has $\|A z\| \leq R$.

Let $z \in \mathcal{P}_{\mathcal{R}}$. Then for each $t \in[0, \infty)$, we have $G(t, s) \geq 0$. Since $f, g_{1}, g_{2}$ are positive functions, $A_{m} z(t) \geq 0, t \in[0, \infty)$. Furthermore, for $t \in[0, \infty)$

$$
\begin{aligned}
\left|\left(A_{m} z\right)(t)\right| \leq & \sup _{z_{1} \in[0, R], z_{2} \in[-R, R]} h\left(z_{1}, z_{2}\right)\left(\int_{\frac{1}{m}}^{\infty} G(s, s) p(s) v(s) d s\right) \\
& +\frac{\varphi(0)}{D} \int_{0}^{\infty} g_{1}(z(s)) \psi(s) d s+\frac{\theta(\infty)}{D} \int_{0}^{\infty} g_{2}(z(s)) \psi(s) d s \\
\leq & \sup _{z_{1} \in[0, R], z_{2} \in[-R, R]} h\left(z_{1}, z_{2}\right)\left(\int_{\frac{1}{m}}^{\infty} G(s, s) p(s) v(s) d s\right) \\
& +\frac{M}{D}\left[g_{1}(R)+g_{2}(R)\right] \int_{0}^{\infty} \psi(s) d s \\
\leq & \sup _{z_{1} \in[0, R], z_{2} \in[-R, R]} h\left(z_{1}, z_{2}\right)\left(\int_{0}^{\infty} G(s, s) p(s) v(s) d s\right)+B(R) \\
\leq & R
\end{aligned}
$$

and

$$
\begin{aligned}
\left|\left(A_{m} z\right)^{\prime}(t)\right| \leq & \int_{\frac{1}{m}}^{\infty}\left|G^{\prime}(t, s)\right| p(s) v(s) h\left(z(s), z^{\prime}(s)\right) d s \\
& +\frac{a_{2}}{D p(t)} \int_{0}^{\infty} g_{1}(z(s)) \psi(s) d s+\frac{a_{1}}{D p(t)} \int_{0}^{\infty} g_{2}(z(s)) \psi(s) d s \\
\leq & \frac{c}{p(t)} \sup _{z_{1} \in[0, R], z_{2} \in[-R, R]} h\left(z_{1}, z_{2}\right) \int_{0}^{\infty} G(s, s) p(s) v(s) d s \\
& +\frac{\max \left\{a_{1}, a_{2}\right\}}{D} \sup _{t \in[0, \infty)} \frac{1}{p(t)}\left[g_{1}(R)+g_{2}(R)\right] \int_{0}^{\infty} \psi(s) d s \\
\leq & \sup _{t \in[0, \infty)} \frac{c}{p(t)}\left[\sup _{z_{1} \in[0, R], z_{2} \in[-R, R]} h\left(z_{1}, z_{2}\right) \int_{0}^{\infty} G(s, s) p(s) v(s) d s\right. \\
& \left.+\frac{M}{D}\left[g_{1}(R)+g_{2}(R)\right] \int_{0}^{\infty} \psi(s) d s\right] \\
\leq & R .
\end{aligned}
$$


Inequalities (3.10) and (3.11) yield that $\left\|A_{m} z\right\| \leq R$. Hence, $A_{m}$ is uniformly bounded. Using the similar proof as (3.2) and (3.3), we can obtain that for any $t, t_{1} \in[0, \infty), z \in P_{R}$,

$$
\left\|A_{m} z(t)-A_{m} z\left(t_{1}\right)\right\| \rightarrow 0 \quad \text { as } t \rightarrow t_{1} .
$$

Thus, $A_{m} P_{R}$ is equicontinuous. It follows from

$$
\begin{aligned}
& \left|\left(A_{m} z\right)(t)-\left(A_{m} z\right)(\infty)\right| \\
& \leq \int_{\frac{1}{m}}^{\infty}|G(t, s)-\bar{G}(s)| p(s) v(s) h\left(z(s), z^{\prime}(s)\right) d s \\
& \quad+\frac{|\varphi(t)-\varphi(\infty)|}{D} \int_{0}^{\infty}\left|g_{1}(z(s))\right| \psi(s) d s \\
& \quad+\frac{|\theta(t)-\theta(\infty)|}{D} \int_{0}^{\infty}\left|g_{2}(z(s))\right| \psi(s) d s \\
& \leq S_{R} \int_{\frac{1}{m}}^{\infty}|G(t, s)-\bar{G}(s)| p(s) v(s) d s \\
& \quad+\frac{1}{D} \max \{|\varphi(t)-\varphi(\infty)|,|\theta(t)-\theta(\infty)|\}\left(T_{R}+T_{R}^{\prime}\right) \int_{0}^{\infty} \psi(s) d s \\
& \rightarrow 0 \text { as } t \rightarrow \infty
\end{aligned}
$$

and

$$
\begin{aligned}
\left|\left(A_{m} z\right)^{\prime}(t)-\left(A_{m} z\right)^{\prime}(\infty)\right| & \\
\leq & \frac{1}{D}\left[\left|\frac{1}{p(t)}-\frac{1}{p(\infty)}\right| \int_{\frac{1}{m}}^{t} a_{2} \theta(s) p(s) v(s) h\left(z(s), z^{\prime}(s)\right) d s\right. \\
& +\left|\frac{1}{p(t)}-\frac{1}{p(\infty)}\right| \int_{t}^{\infty} a_{1} \varphi(s) p(s) v(s) h\left(z(s), z^{\prime}(s)\right) d s \\
& \left.+\max \left\{a_{1}, a_{2}\right\}\left|\frac{1}{p(t)}-\frac{1}{p(\infty)}\right| \int_{0}^{\infty}\left[g_{1}(z(s))+g_{2}(z(s))\right] \psi(s) d s\right] \\
\leq & \frac{1}{D}\left[\left|\frac{1}{p(t)}-\frac{1}{p(\infty)}\right| S_{R} \int_{\frac{1}{m}}^{t} a_{2} \theta(s) p(s) v(s) d s\right. \\
& +\left|\frac{1}{p(t)}-\frac{1}{p(\infty)}\right| S_{R} \int_{t}^{\infty} a_{1} \varphi(s) p(s) v(s) d s \\
& \left.+\max \left\{a_{1}, a_{2}\right\}\left|\frac{1}{p(t)}-\frac{1}{p(\infty)}\right|\left(T_{R}+T_{R}^{\prime}\right) \int_{0}^{\infty} \psi(s) d s\right] \\
\rightarrow & 0 \quad \text { as } t \rightarrow \infty .
\end{aligned}
$$

Therefore, $A_{m} P_{R}$ is equiconvergent. Hence, by Lemma 2.2 and the above discussion, we conclude that for each natural number $m, A_{m}: P \rightarrow P$ is completely continuous.

Finally, observe that

$$
\begin{aligned}
\left|(A z)(t)-\left(A_{m} z\right)(t)\right| & =\left|\int_{0}^{\frac{1}{m}} G(t, s) p(s) f\left(s, z(s), z^{\prime}(s)\right) d s\right| \\
& \leq S_{r_{0}} \int_{0}^{\frac{1}{m}} G(s, s) p(s) v(s) d s<\infty
\end{aligned}
$$


and

$$
\begin{aligned}
\left|(A z)^{\prime}(t)-\left(A_{m} z\right)^{\prime}(t)\right| & =\left|\int_{0}^{\frac{1}{m}} G^{\prime}(t, s) p(s) f\left(s, z(s), z^{\prime}(s)\right) d s\right| \\
& \leq S_{r_{0}} \sup _{t \in[0, \infty)} \frac{c}{p(t)} \int_{0}^{\frac{1}{m}} G(s, s) p(s) v(s) d s<\infty .
\end{aligned}
$$

Hence, inequalities (3.14) and (3.15) imply that $\sup _{t \in[0, \infty)}\left|(A z)(t)-\left(A_{m} z\right)(t)\right|<\infty$ and $\sup _{t \in[0, \infty)}\left|(A z)^{\prime}(t)-\left(A_{m} z\right)^{\prime}(t)\right|<\infty$. Then by the assumption (H4) and the absolute continuity of the integral, we get

$$
\lim _{m \rightarrow \infty} \int_{0}^{\frac{1}{m}} G(t, s) p(s) v(s) d s=0
$$

Therefore, the operator $A: \mathcal{B} \rightarrow \mathcal{B}$ is completely continuous and maps the set $P_{R}$ into itself. Hence, the Schauder fixed point theorem can be applied to obtain a solution of the BVP (1.1), (1.2). The theorem is proved.

Example 3.1 Consider the following boundary value problem:

$$
\begin{aligned}
& \frac{1}{e^{t}}\left(e^{t} z^{\prime}(t)\right)^{\prime}+f\left(t, z(t), z^{\prime}(t)\right)=0, \quad t \in(0, \infty), \\
& z(0)-\lim _{t \rightarrow 0^{+}} e^{t} z^{\prime}(t)=\int_{0}^{\infty} \frac{z(s)}{24} \frac{d s}{1+s^{2}}, \\
& \lim _{t \rightarrow+\infty} e^{t} z^{\prime}(t)=\int_{0}^{\infty} \frac{z(s)}{24} \frac{d s}{1+s^{2}}
\end{aligned}
$$

where $f\left(t, z(t), z^{\prime}(t)\right)=\frac{e^{-2 t}(1+t)}{12 \sqrt{t}}\left(z+\left|z^{\prime}\right|\right), a_{1}=1, a_{2}=0, b_{1}=1, b_{2}=1, p(t)=e^{t}, \psi(s)=\frac{1}{1+s^{2}}$, $g_{1}(z(s))=g_{2}(z(s))=\frac{z(s)}{24}$.

It is clear that $f:(0, \infty) \times[0,+\infty) \times \mathbb{R} \rightarrow(0,+\infty)$ is continuous and singular at $t=0$. Set $v(t)=\frac{e^{-2 t}(1+t)}{\sqrt{t}}$ and $h\left(z, z^{\prime}\right)=\frac{z+\left|z^{\prime}\right|}{12}$, it follows from a direct calculation that $M=2, c=1$, and there exists $R=1$ such that the following inequality holds:

$$
\max \left\{1, \sup _{t \in[0, \infty)} \frac{1}{e^{t}}\right\} \sup _{z_{1} \in[0,1], z_{2} \in[-1,1]} h\left(z_{1}, z_{2}\right)\left(\int_{0}^{\infty} G(s, s) p(s) v(s) d s\right)+B(1) \leq 1
$$

Then by Theorem 3.2, the boundary value problem (3.16)-(3.17) has at least one positive solution.

\section{Existence of at least three positive solutions}

Definition 4.1 Let $\mathcal{B}$ be a Banach space, $\mathcal{P} \subset \mathcal{B}$ be a cone in $\mathcal{B}$. By a concave nonnegative continuous functional $\varphi$ on $\mathcal{P}$, we mean a continuous functional $\varphi: \mathcal{P} \rightarrow[0, \infty)$ with

$$
\varphi(t x+(1-t) y) \geq t \varphi(x)+(1-t) \varphi(y) \quad \text { for all } x, y \in \mathcal{P} \text { and } t \in[0,1]
$$

For $K, L, R>0$ being constants with $\mathcal{P}$ and $\varphi$ as above, let

$$
\mathcal{P}_{K}=\{x \in \mathcal{P}:\|x\|<K\}
$$


and

$$
\mathcal{P}(\varphi, L, K)=\{x \in \mathcal{P}: L \leq \varphi(x),\|x\| \leq K\}
$$

Theorem 4.1 (Leggett-Williams fixed point theorem [12]) Let $\mathcal{B}$ be a Banach space, $\mathcal{P} \subset \mathcal{B}$ be a cone of $\mathcal{B}$, and $R>0$ be a constant. Suppose $A: \overline{\mathcal{P}}_{R} \rightarrow \overline{\mathcal{P}}_{R}$ is a completely continuous operator and $\varphi$ is a nonnegative, continuous, concave functional on $\mathcal{P}$ with $\varphi(y) \leq\|y\|$ for all $y \in \overline{\mathcal{P}}_{R}$. If there exist $r, L$, and $K$ with $0<r<L<K \leq R$ such that the following conditions hold:

(i) $\{y \in \mathcal{P}(\varphi, L, K): \varphi(y)>L\} \neq \emptyset$ and $\varphi(A y)>L$ for all $y \in \mathcal{P}(\varphi, L, K)$;

(ii) $\|A y\|<r$ for all $\|y\| \leq r$;

(iii) $\varphi(A y)>L$ for all $y \in \mathcal{P}(\varphi, L, R)$ with $\|A y\|>K$.

Then $A$ has at least three positive solutions $y_{1}, y_{2}$, and $y_{3}$ in $\overline{\mathcal{P}}_{R}$ satisfying

$$
\left\|y_{1}\right\|<r, \quad y_{2} \in\{y \in \mathcal{P}(\varphi, L, R): \varphi(y)>L\},
$$

and

$$
y_{3} \in \overline{\mathcal{P}}_{R}-\left\{\mathcal{P}(\varphi, L, R) \cup \overline{\mathcal{P}}_{r}\right\}
$$

Theorem 4.2 Assume that (H1)-(H4) are satisfied and there exists $c_{1} \in(0,1)$ such that $m \geq c_{1} M$ holds. Then the boundary value problem (1.1), (1.2) has at least three positive solutions if the following conditions hold:

(H5) There exists a constant $r>0$ such that

$$
h\left(z_{1}, z_{2}\right)<\min \left\{1, \frac{1}{\sup _{t \in[0, \infty)} \frac{c}{p(t)}}\right\} \frac{r-B(r)}{\int_{0}^{\infty} G(s, s) p(s) v(s) d s}
$$

for $t \in[0, \infty)$ and $z_{1} \in[0, r], z_{2} \in[-r, r]$;

(H6) There exist $L>r$ and an interval $[a, b] \subset[0, \infty)$ such that

$$
h\left(z_{1}, z_{2}\right)>\frac{L}{\gamma_{o} k_{0} \int_{0}^{\infty} G(s, s) p(s) v(s) d s},
$$

for $t \in[a, b]$ and $z_{1} \in[L, K], z_{2} \in[-K, K]$;

(H7) There exist $0<r<L<K<\gamma_{0} k_{0} \min \left\{1, \sup _{t \in[0, \infty)} \frac{c}{p(t)}\right\}[R-B(R)], N^{-1} L \leq \max \{1$, $\left.\sup _{t \in[0, \infty)} \frac{c}{p(t)}\right\} K$, where $N=\min \left\{c_{1}, \gamma_{0} k_{0}\right\}$ such that

$$
h\left(z_{1}, z_{2}\right)<\min \left\{1, \frac{1}{\sup _{t \in[0, \infty)} \frac{c}{p(t)}}\right\} \frac{R-B(R)}{\int_{0}^{\infty} G(s, s) p(s) v(s) d s}
$$

for $t \in[0, \infty)$ and $z_{1} \in[0, R], z_{2} \in[-R, R]$.

Proof The conditions of the Leggett-Williams fixed point theorem will be shown to be satisfied. Define the cone $\mathcal{P} \subset B$ by $\mathcal{P}=\{z \in B: z(t) \geq 0$ for each $t \in[0, \infty)\}$ and the nonnegative, continuous, concave functional $\varphi: \mathcal{P} \rightarrow[0, \infty)$ by $\varphi(z)=\min _{t \in[a, b]}|z(t)|$. 
Then we have $\varphi(z) \leq\|z\|$ for all $z \in \mathcal{P}$. If $z \in \overline{\mathcal{P}}_{R}$, then $\|z\| \leq R$ and from (H7) we have

$$
\begin{aligned}
|A z(t)|= & \mid \int_{0}^{\infty} G(t, s) p(s) f\left(s, z(s), z^{\prime}(s)\right) d s+\frac{\varphi(t)}{D} \int_{0}^{\infty} g_{1}(z(s)) \psi(s) d s \\
& +\frac{\theta(t)}{D} \int_{0}^{\infty} g_{2}(z(s)) \psi(s) d s \mid \\
\leq & \int_{0}^{\infty} G(s, s) p(s) v(s) h\left(z(s), z^{\prime}(s)\right) d s+\frac{\varphi(0)}{D} \int_{0}^{\infty} g_{1}(z(s)) \psi(s) d s \\
& +\frac{\theta(\infty)}{D} \int_{0}^{\infty} g_{2}(z(s)) \psi(s) d s \\
\leq & \int_{0}^{\infty} G(s, s) p(s) v(s) h\left(z(s), z^{\prime}(s)\right) d s+\frac{M}{D}\left[g_{1}(R)+g_{2}(R)\right] \int_{0}^{\infty} \psi(s) d s \\
\leq & R .
\end{aligned}
$$

Furthermore,

$$
\begin{aligned}
\left|(A z)^{\prime}(t)\right| \leq & \int_{0}^{\infty}\left|G^{\prime}(t, s)\right| p(s) v(s) h\left(z(s), z^{\prime}(s)\right) d s \\
& +\frac{a_{2}}{D p(t)} \int_{0}^{\infty} g_{1}(z(s)) \psi(s) d s+\frac{a_{1}}{D p(t)} \int_{0}^{\infty} g_{2}(z(s)) \psi(s) d s \\
\leq & \frac{c}{p(t)} \int_{0}^{\infty} G(s, s) p(s) v(s) h\left(z(s), z^{\prime}(s)\right) d s \\
& +\frac{\max \left\{a_{1}, a_{2}\right\}}{D p(t)}\left[g_{1}(R)+g_{2}(R)\right] \int_{0}^{\infty} \psi(s) d s \\
\leq & \sup _{t \in[0, \infty)} \frac{c}{p(t)}\left[\int_{0}^{\infty} G(s, s) p(s) v(s) h\left(z(s), z^{\prime}(s)\right) d s+B(R)\right] \\
\leq & R .
\end{aligned}
$$

Therefore, we get $\|A z\| \leq R$, and this implies that $A: \overline{\mathcal{P}}_{R} \rightarrow \overline{\mathcal{P}}_{R}$.

Now we show that condition (i) of Theorem 4.1 is satisfied. Let $z(t)=\frac{L+K}{2}$ for $t \in[0, \infty)$. By the definition of $\mathcal{P}(\varphi, L, K), z \in \mathcal{P}(\varphi, L, K)$. Then $\{z \in \mathcal{P}(\varphi, L, K): \varphi(z)>L\} \neq \emptyset$. If $z \in$ $\mathcal{P}(\varphi, L, K)$, then by $(\mathrm{H} 6)$ we get

$$
\begin{aligned}
\varphi(A z)= & \min _{t \in[a, b]}\left(\int_{0}^{\infty} G(t, s) p(s) f\left(s, z(s), z^{\prime}(s)\right) d s+\frac{\varphi(t)}{D} \int_{0}^{\infty} g_{1}(z(s)) \psi(s) d s\right. \\
& \left.+\frac{\theta(t)}{D} \int_{0}^{\infty} g_{2}(z(s)) \psi(s) d s\right) \\
\geq & \min _{t \in[a, b]}\left(\int_{0}^{\infty} G(t, s) p(s) f\left(s, z(s), z^{\prime}(s)\right) d s\right) \\
\geq & \gamma_{0} \int_{0}^{\infty} G(s, s) p(s) u(s) h\left(z(s), z^{\prime}(s)\right) d s \\
\geq & \gamma_{0} k_{0} \int_{0}^{\infty} G(s, s) p(s) v(s) h\left(z(s), z^{\prime}(s)\right) d s \\
> & L .
\end{aligned}
$$

Therefore, condition (i) of Theorem 4.1 is satisfied. 
If $\|z\| \leq r$, then by (H5) we have

$$
\begin{aligned}
|A z(t)|= & \mid \int_{0}^{\infty} G(t, s) p(s) f\left(s, z(s), z^{\prime}(s)\right) d s+\frac{\varphi(t)}{D} \int_{0}^{\infty} g_{1}(z(s)) \psi(s) d s \\
& +\frac{\theta(t)}{D} \int_{0}^{\infty} g_{2}(z(s)) \psi(s) d s \mid \\
\leq & \int_{0}^{\infty} G(s, s) p(s) v(s) h\left(z(s), z^{\prime}(s)\right) d s+\frac{\varphi(0)}{D} \int_{0}^{\infty} g_{1}(z(s)) \psi(s) d s \\
& +\frac{\theta(\infty)}{D} \int_{0}^{\infty} g_{2}(z(s)) \psi(s) d s \\
\leq & \int_{0}^{\infty} G(s, s) p(s) v(s) h\left(z(s), z^{\prime}(s)\right) d s+\frac{M}{D}\left[g_{1}(r)+g_{2}(r)\right] \int_{0}^{\infty} \psi(s) d s \\
< & r .
\end{aligned}
$$

In a similar way as (4.1), we can see that for each $t \in[0, \infty)$,

$$
\left|(A z)^{\prime}(t)\right| \leq \sup \frac{c}{p(t)}\left[\int_{0}^{\infty} G(s, s) p(s) v(s) h\left(z(s), z^{\prime}(s)\right) d s+B(r)\right]<r .
$$

Hence, condition (ii) of Theorem 4.1 holds.

Finally, we show that condition (iii) of Theorem 4.1 is also satisfied. If $z \in \mathcal{P}(\varphi, L, R)$, we get

$$
\begin{aligned}
|A z(t)| \leq & \int_{0}^{\infty} G(s, s) p(s) \nu(s) h\left(z(s), z^{\prime}(s)\right) d s+\frac{\varphi(0)}{D} \int_{0}^{\infty} g_{1}(z(s)) \psi(s) d s \\
& +\frac{\theta(\infty)}{D} \int_{0}^{\infty} g_{2}(z(s)) \psi(s) d s \\
\leq & \int_{0}^{\infty} G(s, s) p(s) v(s) h\left(z(s), z^{\prime}(s)\right) d s+\frac{M}{D} \int_{0}^{\infty}\left[g_{1}(z(s))+g_{2}(z(s))\right] \psi(s) d s
\end{aligned}
$$

and

$$
\begin{aligned}
\left|(A z)^{\prime}(t)\right| \leq & \sup \frac{c}{p(t)}\left[\int_{0}^{\infty} G(s, s) p(s) v(s) h\left(z(s), z^{\prime}(s)\right) d s\right. \\
& \left.+\frac{M}{D} \int_{0}^{\infty}\left[g_{1}(z(s))+g_{2}(z(s))\right] \psi(s) d s\right] .
\end{aligned}
$$

Hence, we have

$$
\begin{aligned}
\|A z\| \leq & \max \left\{1, \sup \frac{c}{p(t)}\right\}\left[\int_{0}^{\infty} G(s, s) p(s) v(s) h\left(z(s), z^{\prime}(s)\right) d s\right. \\
& \left.+\frac{M}{D} \int_{0}^{\infty}\left[g_{1}(z(s))+g_{2}(z(s))\right] \psi(s) d s\right] .
\end{aligned}
$$

Therefore, for $z \in \mathcal{P}(\varphi, L, R)$ and $\|A z\|>K$, we have

$$
\begin{aligned}
\varphi(A z)= & \min _{t \in[a, b]}\left(\int_{0}^{\infty} G(t, s) p(s) f\left(s, z(s), z^{\prime}(s)\right) d s+\frac{\varphi(t)}{D} \int_{0}^{\infty} g_{1}(z(s)) \psi(s) d s\right. \\
& \left.+\frac{\theta(t)}{D} \int_{0}^{\infty} g_{2}(z(s)) \psi(s) d s\right)
\end{aligned}
$$




$$
\begin{aligned}
\geq & \gamma_{0} \int_{0}^{\infty} G(s, s) p(s) f\left(s, z(s), z^{\prime}(s)\right) d s+\frac{\varphi(\infty)}{D} \int_{0}^{\infty} g_{1}(z(s)) \psi(s) d s \\
& +\frac{\theta(0)}{D} \int_{0}^{\infty} g_{2}(z(s)) \psi(s) d s \\
\geq & \gamma_{0} \int_{0}^{\infty} G(s, s) p(s) u(s) h\left(z(s), z^{\prime}(s)\right) d s+\frac{m}{D} \int_{0}^{\infty}\left[g_{1}(z(s))+g_{2}(z(s))\right] \psi(s) d s \\
\geq & \gamma_{0} k_{0} \int_{0}^{\infty} G(s, s) p(s) v(s) h\left(z(s), z^{\prime}(s)\right) d s+\frac{c_{1} M}{D} \int_{0}^{\infty}\left[g_{1}(z(s))+g_{2}(z(s))\right] \psi(s) d s \\
\geq & N\left[\int_{0}^{\infty} G(s, s) p(s) v(s) h\left(z(s), z^{\prime}(s)\right) d s+\frac{M}{D} \int_{0}^{\infty}\left[g_{1}(z(s))+g_{2}(z(s))\right] \psi(s) d s\right] \\
\geq & N \frac{N K}{\max \left\{1, \sup _{t \in[0, \infty)} \frac{c}{p(t)}\right\}}\|A z\|>\frac{N}{\max \left\{1, \sup _{t \in[0, \infty)} \frac{c}{p(t)}\right\}} \geq L .
\end{aligned}
$$

Therefore, condition (iii) is also satisfied. Then the Leggett-Williams fixed point theorem implies that $A$ has at least three positive solutions $z_{1}, z_{2}$, and $z_{3}$ which are solutions to the problem (1.1)-(1.2). Furthermore, we have

$$
\left\|z_{1}\right\|<r, \quad z_{2} \in\{z \in \mathcal{P}(\varphi, L, R): \varphi(z)>L\},
$$

and

$$
z_{3} \in \overline{\mathcal{P}}_{R}-\left\{\mathcal{P}(\varphi, L, R) \cup \overline{\mathcal{P}}_{r}\right\}
$$

\section{Competing interests}

The authors declare that they have no competing interests

\section{Authors' contributions}

Both authors typed, read and approved the final manuscript.

Received: 29 June 2012 Accepted: 22 October 2012 Published: 5 November 2012

\section{References}

1. Kosmatov, N: Second order boundary value problems on an unbounded domain. Nonlinear Anal. 68, 875-882 (2008)

2. Lian, $\mathrm{H}, \mathrm{Ge}, \mathrm{W}$ : Existence of positive solutions for Sturm Liouville boundary value problems on the half line. J. Math. Anal. Appl. 321, 781-792 (2006)

3. Tian, Y, Ge, W, Shan, W: Positive solutions for three-point boundary value problem on the half line. Comput. Math. Appl. 53(7), 1029-1039 (2007)

4. Sun, $Y$, Sun, $Y$, Debnath, L: On the existence of positive solutions for singular boundary value problems on the half line. Appl. Math. Lett. 22, 806-812 (2009)

5. Wang, Y, Liu, L, Wu, Y: Positive solutions of singular boundary value problems on the half line. Appl. Math. Comput. 197, 789-796 (2008)

6. Agarwal, RP, O'Regan, D, Wong, PJY: Positive Solutions of Differential, Difference and Integral Equations. Kluwer Academic, Boston (1999)

7. Agarwal, RP, O'Regan, D: Infinite Interval Problems for Differential, Difference and Integral Equations. Kluwer Academic, Dordrecht (2001)

8. Belarbi, A, Benchohra, M, Quahab, A: Multiple positive solutions for nonlinear boundary value problems with integral boundary conditions. Arch. Math. 44, 1-7 (2008)

9. Boucherif, A: Second order boundary value problems with integral boundary conditions. Nonlinear Anal. 70(1), 364-371 (2009)

10. Meiqiang, F: Existence of symmetric positive solutions for a boundary value problem with integral boundary conditions. Appl. Math. Lett. 24(8), 1419-1427 (2011)

11. Zhang, X, Ge, W: Positive solutions for a class of boundary-value problems with integral boundary conditions. Comput. Math. Appl. 58(2), 203-215 (2009)

12. Leggett, RW, Williams, LR: Multiple positive fixed points of nonlinear operators on ordered Banach spaces. Indiana Univ. Math. J. 28, 673-688 (1979)

13. O' Regan, D: Theory of Singular Boundary Value Problem. Word Scientific, Singapore (1994) 
doi:10.1186/1687-2770-2012-127

Cite this article as: Yoruk and Hamal: Existence results for nonlinear boundary value problems with integral boundary conditions on an infinite interval. Boundary Value Problems 2012 2012:127.

Submit your manuscript to a SpringerOpen ${ }^{\circ}$ journal and benefit from:

- Convenient online submission

- Rigorous peer review

- Immediate publication on acceptance

- Open access: articles freely available online

- High visibility within the field

- Retaining the copyright to your article

Submit your next manuscript at $\gg$ springeropen.com 